\title{
Conditional Variance Estimation in Regression Models with Long Memory
}

\author{
Rafał Kulik* Cornelia Wichelhaus $^{\dagger}$
}

October 11, 2011

\begin{abstract}
This paper studies asymptotic properties of a nonparametric kernel estimator of the conditional variance in a random design model with parametric mean and heteroscedastic errors, for a class of long memory errors and predictors. We establish small and large bandwidths asymptotics, which show a different behaviour compared to that of kernel estimators of the conditional mean. We distinguish between an oracle case (i.e., where the errors are directly observed) and a non-oracle case (where the errors are replaced with residuals), and show non-equivalence between the oracle and non-oracle case. We also discuss a practical problem of bandwidth choice. Theoretical results are justified by simulation studies. We apply our theory to DJA and FTSE indices.
\end{abstract}

\section{Introduction}

Consider the random design regression model,

$$
Y_{i}=\beta_{0}+\beta_{1} X_{i}+\sigma\left(X_{i}\right) \varepsilon_{i}, \quad i=1, \ldots, n,
$$

with intercept $\beta_{0}$ and slope $\beta_{1}$. We will study the model (1.1) in the presence of long memory behaviour of the errors and/or predictors. Our goal is to estimate the conditional variance $\sigma^{2}(\cdot)$ in a nonparametric way. To do so, we first estimate $\beta_{0}$ and $\beta_{1}$ by usual least squares estimators. Then, we estimate $\sigma^{2}(\cdot)$ by smoothing residuals with a kernel $K$ and a bandwidth $h$ :

$$
\hat{\sigma}^{2}(x)=\hat{\sigma}_{h}^{2}(x)=\frac{1}{n h \hat{f}_{h}(x)} \sum_{i=1}^{n}\left(Y_{i}-\hat{\beta}_{0}-\hat{\beta}_{1} X_{i}\right)^{2} K\left(\frac{x-X_{i}}{h}\right),
$$

*Department of Mathematics \& Statistics, University of Ottawa, 585 King Edward Avenue, Ottawa ON K1N 6N5, Canada; e-mail: rkulik@uottawa.ca

$\dagger$ Institute of Applied Mathematics, University of Heidelberg, Im Neuenheimer Feld 294, 69120 Heidelberg, Germany; e-mail: wichelhaus@statlab.uni-heidelberg.de 
where

$$
\hat{f}_{h}(x)=\hat{f}_{h, X}(x)=\frac{1}{n h} \sum_{i=1}^{n} K\left(\frac{x-X_{i}}{h}\right) .
$$

The problem of nonparametric estimation of the conditional variance received a lot of attention in the past decade. Most of the work focuses on a fixed design regression model $Y_{i}=m\left(x_{i}\right)+\sigma\left(x_{i}\right) \varepsilon_{i}$, where $x_{i}=i / n$ and $\varepsilon_{i}, i=1, \ldots, n$, are i.i.d. In this set-up it was shown in Wang et al. (2008) that if $m(\cdot)$ is differentiable, then there is no influence of the estimation of $m(\cdot)$ on the minimax rates of convergence for estimation of $\sigma(\cdot)$. In case of random design regression, a similar lack of influence was proven in Fan and Yao (1998) for weakly dependent data. However, very little is known in case of long memory errors and/or predictors. In case of long memory predictors and i.i.d. errors, it was shown in Zhao and $\mathrm{Wu}$ (2008) that there is no influence of estimating the mean, however, this is basically due to their imposed conditions on the bandwidth choice: for a small bandwidth nonparametric estimation behaves as if data were independent. On the other hand, in Guo and Koul (2008) the authors studied the model (1.1) and established a large bandwidth behaviour, i.e. when $h \rightarrow 0$ sufficiently slow.

The goal of this paper is to present the full asymptotic theory for the conditional variance estimation in the model (1.1), when errors and/or predictors have long memory. Such situations are very often encountered in financial time series. We will distinguish between an oracle and a non-oracle case. In the first situation, we assume that $\beta_{0}$ and $\beta_{1}$ are known, which amounts to estimation of $\sigma(\cdot)$ from direct observations $\sigma^{2}\left(X_{i}\right) \varepsilon_{i}^{2}$. In other words, the oracle estimator is defined as

$$
\hat{\sigma}_{\text {oracle }}^{2}(x)=\frac{1}{n h \hat{f}_{h}(x)} \sum_{i=1}^{n} \sigma^{2}\left(X_{i}\right) \varepsilon_{i}^{2} K\left(\frac{x-X_{i}}{h}\right) .
$$

By applying log-transformation, we may see that the problem is similar to nonparametric estimation of a conditional mean. In the latter situation, we refer to Mielniczuk and $\mathrm{Wu}$ (2004) for the most general results. One has to mention that the rates of convergence for the conditional variance differ when compared to the conditional mean, see Remark 3.4. The results for the oracle case are given in Section 3.1.

In Section 3.2 we state the results which show the influence of estimating $\beta_{0}$ and $\beta_{1}$ on estimation of $\sigma(\cdot)$. If the errors $\varepsilon_{i}, i \geq 1$, are i.i.d., the oracle and non-oracle case are the same, regardless whether the predictors are LRD. This agrees with the findings in Fan and Yao (1998). There, the predictors and errors are weakly dependent, and conditional mean is estimated in a nonparametric way. However, if the errors are LRD, there are two additional terms which may contribute to the limiting behavior of the conditional variance estimator. The first one comes from estimating $\beta_{1}$ and disappears if $\mathrm{E}\left[\sigma\left(X_{1}\right)\left(X_{1}-\mu\right)\right]=0$. The second one comes from estimating $\beta_{0}$ and always contributes. 
In Section 3.3 we discuss problem of bandwidth choice. We note that "large" bandwidths are not "practical", since they lead to a complicated LRD-type behaviour. Therefore, it is of practical interest to verify if a chosen bandwidth (for example, by implementing the plug-in method) leads to i.i.d. type behaviour.

We extend results in Guo and Koul (2008) in several directions. First, we establish a "practical", small bandwidth asymptotics. Furthermore, we allow predictors to be follow an EGARCH-type model. This allow us to implement our theory to "typical" financial time series (see Section 4), which cannot be modeled by linear LRD processes. In particular, we apply our theory do Dow Jones Composite Average and FTSE Indices, which have a "typical" behaviour: log-returns are uncorrelated, but residuals have long memory.

We would like to mention that our theory is further developed in Kulik and Wichelhaus (2011). There, we study the limiting behaviour of $\hat{\sigma}_{h}^{2}$, when the conditional mean is estimated in a nonparametric way. Furthermore, we estimate the error density.

\section{Preliminaries}

\subsection{Predictors and the error sequence}

Throughout the paper it is assumed that the predictors $X_{i}, i \geq 1$, are independent of the errors $\varepsilon_{i}, i \geq 1$. We consider the following assumptions on the predictors $X_{i}, i \geq 1$ :

(P1) $X_{i}, i \geq 1$, are i.i.d. random variables with $\mathrm{E} X_{1}=\mu<\infty$ and $\operatorname{Var} X_{1}=1$.

(P2) $X_{i}, i \geq 1$, is EGARCH model defined as $X_{i}=Z_{i} \varphi\left(U_{i}\right), i \geq 1$. Here, $Z_{i}$ is a centered sequence of random variables, $\varphi(\cdot)$ is a real valued function and $U_{i}, i \geq 1$, is a sequence of standard Gaussian random variables with covariance $\mathrm{E}\left(U_{1} U_{k+1}\right)=k^{-\alpha_{U}} L(k)$, where $\alpha_{U} \in(0,1)$ and $L$ is slowly varying at infinity. Then $U_{i}$ can be written as $\sum_{k=1}^{\infty} \tilde{c}_{k} \zeta_{i-k}$. We will assume that $\left(\zeta_{i}, Z_{i}\right), i \geq 1$, are i.i.d., with $\operatorname{Var} Z_{1}=\operatorname{Var} \varphi\left(U_{1}\right)=1$.

In particular, random variables $Z_{i}$ can be degenerated with total mass at 1; in this case $X_{i}=\varphi\left(U_{i}\right)$ is a subordinated Gaussian sequence.

We shall consider similar assumptions on the error sequence:

(E1) $\varepsilon_{i}, i \geq 1$, is a sequence of centered i.i.d. random variables with $\mathrm{E} \varepsilon_{1}^{2}=1$.

(E2) $\varepsilon_{i}, i \geq 1$, is an infinite order moving average

$$
\varepsilon_{i}=\sum_{k=0}^{\infty} c_{k} \eta_{i-k}, \quad \text { with } c_{0}=1,
$$

where $\eta_{i},-\infty<i<\infty$, is a sequence of centered i.i.d. random variables with a finite fourth moment, $\mathrm{E}\left[\varepsilon_{1}^{2}\right]=1$, and for some $\alpha_{\varepsilon} \in(0,1), c_{k} \sim$ $C_{0} k^{-\left(\alpha_{\varepsilon}+1\right) / 2}$ as $k \rightarrow \infty$. 
According to different assumptions, we will apply different techniques. If (E1) and (P2) hold, then we will exploit martingale structure of particular sequences. In this case the normality assumption in (P2) is not necessary in fact. If (E2) and (P1) hold, we will decompose random quantities involved into a martingale part and a long memory part. Under (P2) and (E2), we will exploit normality and Hermite expansions. These different techniques seem to be most efficient.

\subsection{Assumptions on bandwidths and functions}

Let $\kappa_{i}=\int u^{i} K(u) d u$. It is assumed that $K(\cdot)$ is symmetric and positive, and has a bounded support, such that $\kappa_{0}=1, \kappa_{1}=0, \kappa_{2} \neq 0$. It is also assumed that $K(\cdot)$ is bounded and continuous. Denote for future use $K_{h}(\cdot):=K(\cdot / h)$.

For a given random variable $V$, let $f_{V}$ denotes its density. Consider the following assumptions on $f=f_{X}, \sigma$ and the bandwidth $h$.

(D1) $f, \sigma$ are defined on $\mathbb{R}$ with $f, \sigma \in C^{2}(\mathcal{I})$ for some finite interval $\mathcal{I}$, where $C^{2}$ is the class of twice-differentiable functions, with bounded and continuous second order derivatives. Also, $\inf _{x \in \mathcal{I}} f(x)>0$.

(D2) $\sigma(x)>0$ for all $x \in \mathbb{R}$ and $\mathrm{E}\left[\sigma^{4}(X)\right]<\infty$.

(D3) $h+\sqrt{n h} h^{2} \rightarrow 0, n h \rightarrow \infty$.

\subsection{Long memory Gaussian sequences}

To introduce further assumptions, let us recall some facts on LRD sequences and Hermite coefficients. A function $G(\cdot)$ in $L^{2}(\phi), \phi(x)=(2 \pi)^{-1 / 2} \exp \left(-x^{2} / 2\right)$, such that $\mathrm{E}\left[G\left(U_{1}\right)\right]=0$, can be expanded as

$$
G(\cdot)=\sum_{m=1}^{\infty} \frac{J_{m}}{m !} H_{m}(\cdot)
$$

where $J_{m}=\mathrm{E}\left(G\left(U_{1}\right) H_{m}\left(U_{1}\right)\right)$ and $H_{m}(\cdot)$ is the $m$ th Hermite polynomial. The smallest $q \geq 1$ such that $J_{q} \neq 0$ is called the Hermite rank of $G$. In particular, let $J_{m}(x)=\mathrm{E}\left[1_{\left\{\varphi\left(U_{1}\right) \leq x\right\}} H_{m}\left(U_{1}\right)\right]$. Then

$$
1_{\{\varphi(\cdot) \leq x\}}-F(x)=\sum_{m=q(x)}^{\infty} J_{m}(x) \frac{H_{m}(\cdot)}{m !},
$$

with some $q(x) \in \mathbb{N}$. Let $q$ be the Hermite rank of class of functions $1_{\{\varphi(\cdot) \leq x\}}-$ $F(x), x \in \mathbb{R}$, i.e.

$$
q=\min \left\{q(x): J_{q(x)}(x) \neq 0 \text { for some } x \in \mathbb{R}\right\} .
$$

The corresponding coefficient is denoted by $J_{q}(x)$. We shall assume that

(J1) $J_{q}^{\prime}(\cdot)$ is uniformly bounded and uniformly continuous. 
(J2) The following function is in $L^{2}(\phi)$ :

$$
e(\cdot ; x) \rightarrow \frac{1}{\varphi(\cdot)} f_{Z}\left(\frac{x}{\varphi(\cdot)}\right) .
$$

Remark 2.1. Condition (J1) is related to the Gaussian sequence $U_{i}$ and the function $\varphi$. It is imposed in Csörgő and Mielniczuk (1995) in case of density estimation for subordinated Gaussian sequences. It is fulfilled for example when $\varphi(u)=u$, since then $q=1, J_{1}(x)=\int_{-\infty}^{x} s \phi(s) d s$, and $J_{1}^{\prime}(x)=x \phi(x)$.

Assumption (J2) links the Gaussian sequence $U_{i}$, the function $\varphi$ and random variables $Z_{i}$. In particular, (J2) implies that quantities

$$
J_{m}^{*}(x):=\mathrm{E}\left[J_{m}^{\prime}\left(\frac{x}{Z_{1}}\right) \frac{1}{Z_{1}}\right]
$$

are well defined for all $m \geq q$.

It is clearly fulfilled if $f_{Z}$ is degenerated, i.e. $X_{i}$ is subordinated Gaussian sequence. Furthermore, if $\varphi(u)=u$ and $f_{Z}$ does not vanish at 0 , then (2.2) is equivalent to $\mathrm{E}\left[U^{-2}\right]<\infty$. However, the latter is not true. Thus, $x=0$ has to be excluded from our analysis. On the other hand, if $f_{Z}$ is bounded with bounded support, say, $(-M, M)$, then for $x \in(-M, M) \backslash\{0\}$,

$$
\mathrm{E}\left[U^{-2} f_{Z}^{2}\left(\frac{x}{U}\right)\right] \leq C \frac{1}{x^{2} M^{2}} \leq \frac{C}{M^{4}} .
$$

Furthermore, if $f_{Z}$ is the standard normal density, then (J2) is fulfilled. Indeed, as $u \rightarrow 0, u^{-2} f_{Z}^{2}(x / u) \sim C u^{-2} \exp \left(-(x / u)^{4} / 2\right) \rightarrow 0$, so that $u^{-2} f_{Z}^{2}(x / u) \phi(u)$ is integrable.

Further comments on (J2) and its relation to (J1) are given in Appendix.

Let us also recall that for if $q \alpha_{U}<1$,

$$
\frac{1}{a_{n, q}} \sum_{j=1}^{n} \frac{1}{q !} H_{q}\left(U_{i}\right) \stackrel{\mathrm{d}}{\rightarrow} L_{q}
$$

where $a_{n, q}^{2} \sim n^{2-q \alpha_{U}} L^{q}(n) / C^{2}\left(q, \alpha_{U}\right), C\left(q, \alpha_{U}\right)=\sqrt{\left(1-q \alpha_{U}\right)\left(2-q \alpha_{U}\right) /(2 q !)}$,

$$
L_{q}=\left(q !\left(1-q \alpha_{U}\right)^{-1 / 2} Z_{q}(1)\right.
$$

and $Z_{q}(t)$ is the so-called Hermite or Rosenblatt process of order $q$, defined as a $q$-fold stochastic integral

$$
Z_{q}(t)=\int_{-\infty}^{\infty} \ldots \int_{-\infty}^{\infty} \frac{\mathrm{e}^{\mathrm{i} t\left(x_{1}+\cdots+x_{q}\right)}-1}{x_{1}+\cdots+x_{q}} \prod_{i=1}^{q} x_{i}^{\left(1-\alpha_{U}\right) / 2} W\left(d x_{1}\right) \ldots W\left(d x_{q}\right),
$$

where $W$ is an independently scattered Gaussian random measure with Lebesgue control measure. If $q \alpha_{U}>1$, then $\sum_{i=1}^{n} H_{q}\left(U_{i}\right)=O_{P}(\sqrt{n})$.

For more details on Gaussian LRD sequences the reader is referred to Taqqu (2003). 


\subsection{Limit theorems for long memory linear processes}

First, let us introduce the following $\sigma$-fields: let $\mathcal{X}_{i}$ be the $\sigma$-field generated by $\left(Z_{i}, \zeta_{i-1}, Z_{i-1}, \zeta_{i-2} \ldots\right)$ and let $\mathcal{H}_{i}=\sigma\left(\eta_{i}, \eta_{i-1}, \ldots\right)$. Under the assumption (E2) we have

$$
b_{n}^{2}:=\operatorname{Var}\left(\sum_{i=1}^{n} \varepsilon_{i}\right) \sim C_{1}^{2} n^{2-\alpha_{\varepsilon}}, \quad \alpha_{\varepsilon} \in(0,1),
$$

with

$$
C_{1}^{2}=\frac{2 C_{0}^{2}}{\left(1-\alpha_{\varepsilon}\right)\left(2-\alpha_{\varepsilon}\right)} \int_{0}^{\infty}\left(x+x^{2}\right)^{-\left(\alpha_{\varepsilon}+1\right) / 2} d x .
$$

Furthermore, (see Davydov (1970))

$$
b_{n}^{-1} \sum_{i=1}^{n} \varepsilon_{i} \stackrel{\mathrm{d}}{\rightarrow} \mathcal{N}(0,1) .
$$

Let $\xi_{i}=\varepsilon_{i}^{2}-\mathrm{E}\left[\varepsilon_{i}^{2}\right]=\varepsilon_{i}^{2}-1$. One can verify that with some $C>0$,

$$
\operatorname{Cov}\left(\xi_{0}, \xi_{i}\right) \sim C^{2} \operatorname{Cov}^{2}\left(\varepsilon_{0}, \varepsilon_{j}\right),
$$

see e.g. Guo and Koul (2008, Lemma 4.1). Thus, for $d_{n}^{2}:=\operatorname{Var}\left(\sum_{i=1}^{n} \xi_{i}\right)$, we have

$$
d_{n}^{2} \sim \begin{cases}C_{2}^{2} n^{2\left(1-\alpha_{\varepsilon}\right)}, & \text { if } \alpha_{\varepsilon}<1 / 2, \\ C_{3}^{2} n, & \text { if } \alpha_{\varepsilon}>1 / 2,\end{cases}
$$

with some constants $C_{2}, C_{3}$. Also,

$$
\operatorname{Var}\left(\sum_{i=1}^{n} \varepsilon_{i}^{2}\right) \sim \begin{cases}C_{2}^{2} n^{2\left(1-\alpha_{\varepsilon}\right)}, & \text { if } \alpha_{\varepsilon}<1 / 2 \\ C_{4}^{2} n, & \text { if } \alpha_{\varepsilon}>1 / 2,\end{cases}
$$

with a possibly different constant $C_{4}$. Moreover, (see Avram and Taqqu (1987, Theorem 2),

$$
d_{n}^{-1} \sum_{i=1}^{n} \xi_{i} \stackrel{\mathrm{d}}{\rightarrow} Z_{2}(1), \quad d_{n}^{-1} \sum_{i=1}^{n} \mathrm{E}\left[\xi_{i} \mid \mathcal{H}_{i-1}\right] \stackrel{\mathrm{d}}{\rightarrow} Z_{2}(1), \quad \text { if } \alpha_{\varepsilon}<1 / 2,
$$

where $\mathrm{H}_{2}$ is a Hermite-Rosenblatt random variable. If $\alpha_{\varepsilon}>1 / 2$, then the above limits are standard normal with $\sqrt{n}$-normalization.

\section{Results}

\subsection{Conditional variance: oracle case}

Here, we consider behaviour of the oracle estimator defined in (1.4). 
Proposition 3.1. Assume (E1) and either (P2) or (P1). Suppose that assumptions of Section 2.2 and (J1)-(J2) are fulfilled. Then

$$
\sqrt{n h f(x)}\left(\hat{\sigma}_{\text {oracle }}^{2}(x)-\sigma^{2}(x)\right) \stackrel{d}{\rightarrow} \mathcal{N}\left(0, \sigma^{4}(x)\left(\mathrm{E}\left[\left(\varepsilon_{1}^{2}-1\right)^{2}\right]\right) \int K^{2}\right) .
$$

Proposition 3.2. Assume (E2) and either (P2) or (P1). Suppose that assumptions of Section 2.2 and (J1)-(J2) are fulfilled. We have:

- If $h n^{\left(1-2 \alpha_{\varepsilon}\right)} \rightarrow 0$, then (3.1) holds.

- If $h n^{\left(1-2 \alpha_{\varepsilon}\right)} \rightarrow \infty$, then

$$
n^{\alpha_{\varepsilon}}\left(\hat{\sigma}_{\text {oracle }}^{2}(x)-\sigma^{2}(x)\right) \stackrel{d}{\rightarrow} C_{2} \sigma^{2}(x) Z_{2}(1),
$$

where the constant $C_{2}$ and random variable $Z_{2}(1)$ are defined in (2.10) and (2.11), respectively.

Remark 3.3. In Proposition 3.1 there is no influence of LRD in predictors. It is in contrast with results in Guo and Koul (2008). The reason for this is that we consider $\hat{f}_{h}$ in the definition of $\hat{\sigma}_{h}^{2}$ instead of $f$ (or $\tilde{f}$, i.e. Gaussian density with estimated mean and variance). This effect is explained in details in Remark 5.2.

Remark 3.4. If $\alpha_{\varepsilon} \in(1 / 2,1)$ there is no influence of LRD in errors on the rates of convergence, which is basically due to (2.9) below. If $\alpha_{\varepsilon} \in(0,1 / 2)$ and $h$ is small (i.e., when $h n^{\left(1-2 \alpha_{\varepsilon}\right)} \rightarrow 0$ holds), then there is still no influence of LRD. However, if $h$ is $b i g$ (i.e., when $h n^{\left(1-2 \alpha_{\varepsilon}\right)} \rightarrow \infty$ holds), LRD influences the limit. Note further that the meaning of small and big bandwidth is different than in case of estimating conditional mean in a nonparametric way. Namely, in the model $Y_{i}=m\left(X_{i}\right)+\sigma\left(X_{i}\right) \varepsilon_{i}$, for the standard kernel estimator of $m(\cdot)$, we have $\sqrt{n h}$ or $n^{\alpha_{\varepsilon} / 2}$ rate of convergence if, respectively, $h n^{\left(1-\alpha_{\varepsilon}\right)} \rightarrow 0$ or $h n^{\left(1-\alpha_{\varepsilon}\right)} \rightarrow \infty$. We refer to Mielniczuk and Wu (2004) for more details.

\subsection{Conditional variance: non-oracle case}

In the non-oracle case, i.e., when $\beta_{0}, \beta_{1}$ are unknown and have to be estimated, the conditional variance is estimated using the kernel estimator (1.2). We will focus on cases (E1) and (E2)+(P1), to show an influence of LRD in the errors. The case of $(\mathrm{E} 2)+(\mathrm{P} 2)$ is skipped here. It is a subject of Kulik and Wichelhaus (2011) in a more general context of nonparametric conditional mean.

Theorem 3.5. Assume (E1) and either $(\mathrm{P} 2)$ or $(\mathrm{P} 1)$. Suppose that assumptions of Section 2.2 and (J1)-(J2) are fulfilled. Then

$$
\sqrt{\operatorname{nhf}(x)}\left(\hat{\sigma}^{2}(x)-\sigma^{2}(x)\right) \stackrel{d}{\rightarrow} \mathcal{N}\left(0, \sigma^{4}(x)\left(\mathrm{E}\left[\left(\varepsilon_{1}^{2}-1\right)^{2}\right]\right) \int K^{2}\right) .
$$


Theorem 3.6. Assume (E2) and (P1). Suppose that assumptions of Section 2.2 and (J1)-(J2) are fulfilled.

- If $h n^{\left(1-2 \alpha_{\varepsilon}\right)} \rightarrow 0$, then (3.3) holds.

- If $h n^{\left(1-2 \alpha_{\varepsilon}\right)} \rightarrow \infty$, then

$$
\begin{aligned}
& \left.n^{\alpha_{\varepsilon}}\left(\hat{\sigma}^{2}(x)-\sigma^{2}(x)\right]\right) \stackrel{d}{\rightarrow} C_{2} \sigma^{2}(x) Z_{2}(1) \\
& +C_{1}^{2}\left(\mathrm{E}^{2}\left[\sigma\left(X_{1}\right)\left(X_{1}-\mu\right)\right](x-\mu)^{2}-2 \sigma(x)(x-\mu) \mathrm{E}\left[\sigma\left(X_{1}\right)\left(X_{1}-\mu\right)\right]\right) \chi^{2}(1) \\
& +C_{1}^{2}\left(\mathrm{E}^{2}\left[\sigma\left(X_{1}\right)\right]-2 \sigma(x) \mathrm{E}\left[\sigma\left(X_{1}\right)\right]\right) \chi^{2}(1)
\end{aligned}
$$

where $\chi^{2}(1)$ is $\chi^{2}$ random variable with 1 degree of freedom.

Remark 3.7. Comparing with Proposition 3.2, Theorem 3.6 reveals the influence of estimation of the linear regression parameters, when errors are LRD. If $\alpha_{\varepsilon}>1 / 2$, then there is no difference between oracle and non-oracle case, and in fact the results are as in the case of i.i.d. errors. Second, there are two additional $O_{P}\left(n^{-\alpha_{\varepsilon}}\right)$ terms in (3.4). The first one comes from estimating $\beta_{1}$ and vanishes if $\mathrm{E}\left[\sigma\left(X_{1}\right)\left(X_{1}-\mu\right)\right]=0$. The second one comes from estimating $\beta_{0}$.

Remark 3.8. Once again, let us compare Theorem 3.6 with Guo and Koul (2008, Theorem 3.1), by setting $\alpha_{X}=1$ there. Part (a) there is not applicable. Results in Part (b) there agree with (3.4) of Theorem 3.6.

Remark 3.9. The results of Propositions 3.1, 3.2 and Theorems 3.5, 3.6 can be formulated in a multivariate set-up. In case of (3.1) the limiting distribution of $\left(\hat{\sigma}^{2}\left(x_{i}\right)-\sigma^{2}\left(x_{i}\right), i=1, \ldots, m\right)$ is asymptotically multivariate normal with independent components (it follows using the Cramer-Wold device). In case of (3.2) the limiting distribution is degenerate,

$$
C_{2}\left(\sigma^{2}\left(x_{i}\right), i=1, \ldots, m\right) Z_{2}(1),
$$

in case of (3.4) the limiting distribution is also degenerate.

\subsection{Bandwidth choice}

Let us note first that from a practical point of view "large" bandwidths are not desirable. Indeed, in the context of (3.4) it is completely not clear how to estimate percentiles of the limiting distribution, since e.g. bootstrap does not work (see Lahiri (2003, Chapter 10)). Therefore, results in LRD zone (i.e., the results in (3.2) and (3.4)) are not practical and may serve as a warning against inappropriate choice of $h$.

In Kulik and Lorek (2011) the authors studied the problem of bandwidth choice for the conditional mean estimation. It was proven there that crossvalidation may not be a valid procedure in case of long memory errors, and plug-in methods are preferable. In Section 4 we justify the latter statement 
Table 1: Bandwidth choice for $\sigma(\cdot)$

\begin{tabular}{||c||c|ccccc||}
\hline \hline$d_{\varepsilon}$ & & $q_{0.05}$ & $q_{0.25}$ & $q_{0.5}$ & $q_{0.75}$ & $q_{0.95}$ \\
\hline \hline 0 & $\hat{h}$ & 0.1108 & 0.1725 & 0.2346 & 0.2639 & 0.2960 \\
0.1 & $\hat{h}$ & 0.1124 & 0.1725 & 0.2336 & 0.2660 & 0.2990 \\
0.2 & $\hat{h}$ & 0.1014 & 0.1828 & 0.2332 & 0.2666 & 0.3037 \\
0.3 & $\hat{h}$ & 0.1141 & 0.1750 & 0.2318 & 0.2633 & 0.2980 \\
0.4 & $\hat{h}$ & 0.1025 & 0.1754 & 0.2307 & 0.2613 & 0.2952 \\
\hline \hline
\end{tabular}

based on simulation studies. It is shown that there is little influence of LRD in errors on the plug-in bandwidth choice. Thus, in our implementation in Section 4 we apply the procedure:

- Estimate $h$ using the plug-in method. Solve $n^{-\delta}=h$. Compare the obtained $\delta$ with $1-2 \hat{\alpha}_{\varepsilon}$, where $\hat{\alpha}_{\varepsilon}$ is an estimator of $\alpha_{\varepsilon}$; for example method from Guo and Koul (2008) leads to a consistent estimator. Verify if $h n^{1-2 \alpha_{\varepsilon}} \rightarrow 0$ holds. In particular, there is nothing to verify if $\alpha_{\varepsilon}>1 / 2$.

In this way the estimator of $\alpha_{\varepsilon}$ is not used to construct confidence intervals, rather to justify if we can use (3.1) to construct confidence intervals.

\section{Numerical studies and data examples}

\subsection{Simulation studies}

- We simulate $n=1000$ observations from the models $Y_{i}=0+2 X_{i}+\sigma\left(X_{i}\right) \varepsilon_{i}$, with $\sigma(x)=\sqrt{x^{2}+1}$ the predictors $X_{i}$ are i.i.d. Gaussian and the errors $\varepsilon_{i}$ are Gaussian FARIMA $\left(0, d_{\varepsilon}, 0\right)$. Here, $d_{\varepsilon}=\left(1-\alpha_{\varepsilon}\right) / 2 \in(0,1 / 2)$.

- We estimate $\beta_{0}$ and $\beta_{1}$ by LSE estimators. We compute the estimator $\hat{\sigma}_{\hat{h}}^{2}(\cdot)$ with $\hat{h}$ selected by the plug-in method.

- This procedure is repeated $M=500$ times.

- Table 1 contains statistics for the bandwidth $h$ selected by the plug-in method. There is little influence of the memory parameter.

\subsection{Data Analysis}

We study regression relationship between Dow Jones Composite Average $(Y)$ and FTSE Index (predictor) from 1 January 2000 to 1 January 2010 (source: Yahoo Finance). From figure below we conclude that there is no correlation in log-returns, however, there is a strong correlation in squared log-returns. The memory parameter for the latter is estimated to be $d=0.17$. Also, normality of the predictor can be assumed. Finally, the conditional variance is plotted. The bandwidth for the conditional variance was chosen using plug-in method. 
Also, there is a little memory in residuals. This means that pointwise confidence intervals can be constructed using (3.1).

\section{Proofs}

\subsection{Density estimation for SV models}

Asymptotic behaviour of the kernel density estimator is well known in case of subordinated Gaussian or linear processes with LRD, see e.g. Csörgö and Mielniczuk (1995), Wu and Mielniczuk (2002). Here, we establish it under (P2). Consistency is needed later.

Lemma 5.1. Suppose that assumptions of Section 2.2 and (J1)-(J2) are fulfilled.

- If $h a_{n, q}^{2} / n \rightarrow 0$, then

$$
\sqrt{n h}\left(\hat{f}_{h}(x)-f(x)\right) \stackrel{d}{\rightarrow} \mathcal{N}\left(0, f(x) \int K^{2}(u) d u\right) .
$$

- If $h a_{n, q}^{2} / n \rightarrow \infty$, then

$$
\frac{n}{a_{n, q}}\left(\hat{f}_{h}(x)-f(x)\right) \stackrel{d}{\rightarrow} J_{q}^{*}(x) L_{q}
$$

Proof. We decompose

$$
\begin{aligned}
& \frac{1}{n h} \sum_{i=1}^{n}\left\{K_{h}\left(x-X_{i}\right)-f(x)\right\}=\left\{h^{-1} \mathrm{E}\left[K_{h}\left(x-X_{1}\right)\right]-f(x)\right\} \\
& \quad+\frac{1}{n h} \sum_{i=1}^{n}\left\{K_{h}\left(x-X_{i}\right)-\mathrm{E}\left[K_{h}\left(x-X_{i}\right) \mid \mathcal{X}_{i-1}\right]\right\}+ \\
& +\frac{1}{n h} \sum_{i=1}^{n}\left\{\mathrm{E}\left[K_{h}\left(x-X_{i}\right) \mid \mathcal{X}_{i-1}\right]-\mathrm{E}\left[K_{h}\left(x-X_{i}\right)\right]\right\}=: A_{1}+A_{2}+A_{3} .
\end{aligned}
$$

The first part in the above decomposition is the bias and is of order $h^{2}$, due to assumptions on $K$ and $f$. The second part is a martingale, so that from martingale CLT (see Appendix) we conclude

$$
\sqrt{n h} A_{2} \stackrel{\mathrm{d}}{\rightarrow} \mathcal{N}\left(0, f(x) \int K^{2}(u) d u\right)
$$

It remains to deal with LRD part $A_{3}$. Since $K$ is bounded, a function

$$
(\cdot) \rightarrow \int K_{k}(x-z \varphi(\cdot)) f_{Z}(z) d z
$$


is in $L^{2}(\phi)$. Applying the Hermite expansion,

$$
\begin{aligned}
& \frac{1}{n h} \sum_{i=1}^{n}\left\{\mathrm{E}\left[K_{h}\left(x-X_{i}\right) \mid \mathcal{X}_{i-1}\right]-\mathrm{E}\left[K_{h}\left(x-X_{i}\right)\right]\right\} \\
& \quad=\int \frac{1}{n h} \sum_{i=1}^{n}\left\{K_{h}\left(x-z \varphi\left(U_{i}\right)\right)-\mathrm{E}\left[K_{h}\left(x-z \varphi\left(U_{i}\right)\right)\right]\right\} f_{Z}(z) d z \\
& \quad=: \quad \int A_{3}(x ; z) f_{Z}(z) d z .
\end{aligned}
$$

The next few steps are similar to Csörgö and Mielniczuk (1995). Let $\hat{F}_{n}(\cdot)$ be the empirical distribution function associated with random variables $\varphi\left(U_{1}\right), \ldots, \varphi\left(U_{n}\right)$. Define the corresponding empirical processes

$$
t_{n}(y)=\frac{n}{a_{n, q}}\left(\hat{F}_{n}(y)-F(y)\right), \quad \tilde{t}_{n}(y)=t_{n}(y)-J_{q}(y) \frac{1}{a_{n, q}} \sum_{i=1}^{n} \frac{1}{m !} H_{m}\left(U_{i}\right) .
$$

Then

$$
\begin{aligned}
& \frac{n}{a_{n, q}} A_{3}(x ; z)=\frac{1}{h} \int K\left(\frac{x-z y}{h}\right) d t_{n}(y)=\frac{z}{h^{2}} \int t_{n}(y) K^{\prime}\left(\frac{x-z y}{h}\right) d y \\
& \quad=\frac{1}{h} \int \tilde{t}_{n}\left(\frac{x-h u}{z}\right) K^{\prime}(u) d u+\frac{1}{h} \frac{1}{a_{n, q}} \sum_{i=1}^{n} \frac{H_{q}\left(U_{i}\right)}{q !} \int J_{q}\left(\frac{x-h u}{z}\right) K^{\prime}(u) d u
\end{aligned}
$$

As in Csörgő and Mielniczuk (1995), the first part converges almost surely to 0 (uniformly in $z, x$ ). Thus

$$
\begin{aligned}
\sup _{x} \mid & \frac{n}{a_{n, q}} \int A_{3}(x ; z) f_{Z}(z) d v \\
& -\frac{1}{a_{n, q}} \sum_{i=1}^{n} \frac{H_{q}\left(U_{i}\right)}{q !} \frac{1}{h} \iint J_{q}\left(\frac{x-h u}{z}\right) K^{\prime}(u) f_{Z}(z) d u d z \mid=o_{P}(1) .
\end{aligned}
$$

Noting that

$$
\begin{aligned}
& \frac{1}{h} \iint J_{q}\left(\frac{x-h u}{z}\right) K^{\prime}(u) f_{Z}(z) d u d z \\
& \quad=\frac{1}{h} \int K(u) J_{q}^{\prime}\left(\frac{x-h u}{z}\right) f_{Z}(z) d u d z \sim \int \frac{1}{z} J_{q}^{\prime}(x / z) f_{Z}(z) d z=J_{q}^{*}(x),
\end{aligned}
$$

the result follows by (2.3) and assumption (J2). 


\subsection{Decomposition of the conditional variance estimator}

For $i \geq 1$, let $\Delta_{i}=\left(\hat{\beta}_{0}-\beta_{0}\right)+\left(\hat{\beta}_{1}-\beta_{1}\right) X_{i}=: \Delta_{0}+\Delta_{1, i}$. Decompose

$$
\begin{aligned}
\left\{\hat{\sigma}^{2}(x)\right. & \left.-\sigma^{2}(x)\right\}=\left(\frac{1}{n h \hat{f}_{h}(x)} \sum_{i=1}^{n} \sigma^{2}\left(X_{i}\right) K_{h}\left(x-X_{i}\right)-\sigma^{2}(x)\right) \\
& +\frac{1}{n h \hat{f}_{h}(x)} \sum_{i=1}^{n} \sigma^{2}\left(X_{i}\right) K_{h}\left(x-X_{i}\right)\left(\varepsilon_{i}^{2}-1\right) \\
& \quad-\frac{2}{n h \hat{f}_{h}(x)} \sum_{i=1}^{n} \Delta_{i} \sigma\left(X_{i}\right) K_{h}\left(x-X_{i}\right) \varepsilon_{i}+\frac{1}{n h \hat{f}_{h}(x)} \sum_{i=1}^{n} \Delta_{i}^{2} K_{h}\left(x-X_{i}\right) \\
=: & M_{1}+M_{2}-M_{3}+M_{4} .
\end{aligned}
$$

In the oracle case, $\Delta_{i}=0$ and thus, $M_{3}=M_{4} \equiv 0$. Therefore, for the oracle estimator we have the following decomposition:

$$
\left\{\hat{\sigma}_{\text {oracle }}^{2}(x)-\sigma^{2}(x)\right\}=M_{1}+M_{2} .
$$

It will be shown that under assumption (D3),

$$
\sqrt{n h} M_{1}=o_{P}(1) .
$$

Then, we will deal with the term $M_{2}$. Under (E1), it behaves as follows:

$$
\sqrt{n h f(x)} M_{2} \stackrel{\mathrm{d}}{\rightarrow} \mathcal{N}\left(0, \sigma^{4}(x) \mathrm{E}\left[\xi_{1}^{2}\right] \int K^{2}\right) .
$$

Under (E2), (5.6) holds if $h n^{\left(1-2 \alpha_{\varepsilon}\right)} \rightarrow 0$. If however (E2)+(P1) holds and $h n^{\left(1-2 \alpha_{\varepsilon}\right)} \rightarrow \infty$,

$$
n^{\alpha_{\varepsilon}} M_{2} \stackrel{\mathrm{d}}{\rightarrow} C_{2} \sigma^{2}(x) Z_{2}(1) .
$$

If $(\mathrm{E} 2)+(\mathrm{P} 2)$ holds and $h n^{\left(1-2 \alpha_{\varepsilon}\right)} \rightarrow \infty$,

$$
n^{\alpha_{\varepsilon}} M_{2} \stackrel{\mathrm{d}}{\rightarrow} C_{2} \sigma^{2}(x) \frac{J_{0}^{*}(x)}{f(x)} Z_{2}(1) .
$$

The decomposition (5.4) together with asymptotic results (5.5)-(5.8) yield Propositions 3.1-3.2.

In the non-oracle case, under (E1) (for both (P1) and (P2)) the terms $M_{3}$ and $M_{4}$ are negligible. Under (E2) and (P1), we show that they contribute as follows:

$$
\begin{aligned}
& n^{\alpha_{\varepsilon}} M_{3} \stackrel{\mathrm{d}}{\rightarrow} 2 C_{1}^{2} \sigma(x)\left(\mathrm{E}\left[\sigma\left(X_{1}\right)\left(X_{1}-\mu\right)\right](x-\mu)+\mathrm{E}\left[\sigma\left(X_{1}\right)\right]\right) \times \chi^{2}(1), \\
& n^{\alpha_{\varepsilon}} M_{4} \stackrel{\mathrm{d}}{\rightarrow} C_{1}^{2} \mathrm{E}^{2}\left[\sigma\left(X_{1}\right)\right] \chi^{2}(1)+(x-\mu)^{2} \omega^{2} \chi^{2}(1),
\end{aligned}
$$


where $\omega^{2}:=C_{1}^{2} \mathrm{E}^{2}\left[\sigma\left(X_{1}\right)\left(X_{1}-\mu\right)\right]$. Since the convergence in (5.9) and (5.10) is joint, these together with (5.5)-(5.8) and the decomposition (5.3) of the variance estimator, will imply the results of Theorem 3.5 and Theorem 3.6.

It is worth to point out that asymptotic behaviour of terms $M_{1}, M_{2}, M_{3}, M_{4}$ is not of particular use, unless we consider them as part of decompositions (5.3)-(5.4). The same apply to terms in different decompositions below.

\subsection{Proof of Propositions 3.1 and 3.2}

As noted above (see (5.4)), in order to prove Propositions 3.1 and 3.2 it is enough to deal with terms $M_{1}$ and $M_{2}$ (see (5.5) and (5.6), respectively). Write $M_{1}$ as

$$
\begin{aligned}
M_{1} & =\left(\frac{1}{\hat{f}_{h}(x)}-\frac{1}{f(x)}\right) \frac{1}{n h} \sum_{i=1}^{n}\left(\sigma^{2}\left(X_{i}\right)-\sigma^{2}(x)\right) K_{h}\left(x-X_{i}\right) \\
& +\frac{1}{n h f(x)} \sum_{i=1}^{n}\left(\sigma^{2}\left(X_{i}\right)-\sigma^{2}(x)\right) K_{h}\left(x-X_{i}\right):=\left(\frac{1}{\hat{f}_{h}(x)}-\frac{1}{f(x)}\right) M_{11}+M_{11} .
\end{aligned}
$$

By Lemma 5.1, $\hat{f}_{h}$ is consistent estimator of $f$. Therefore, the first part in the expression above is dominated by the second one. Since (D1) holds, we have $\mathrm{E}\left|M_{11}\right|=O\left(h^{2}\right)$. Therefore, $\sqrt{n h} M_{1}=o_{P}(1)$ by (D3) and (5.5) follows.

Next, we deal with $M_{2}$. We will prove (5.6), (5.7) and (5.8) under different sets of assumptions. In order to do this, we consider $\hat{f}_{h}$ replaced with $f$. This is allowed, since $\hat{f}_{h}$ is the weakly consistent estimator of $f$, and $K$ has finite support.

Under (E1), $M_{2}$ is a martingale w.r.t. $\mathcal{G}_{n}, n \geq 1$, where $\mathcal{G}_{n}$ is a sigma field generated by $\left(Z_{i}, \eta_{i}, \zeta_{i-1}, Z_{i-1}, \eta_{i-1}, \ldots\right)$. Hence, from martingale CLT (see Appendix) we conclude that (5.6) holds.

Assume now (E2) and (P1). Recall that $\xi_{i}=\varepsilon_{i}^{2}-1$ and define $\mathcal{H}_{i}$ to be a sigma field generated by $\left(\eta_{i}, \eta_{i-1}, \ldots\right)$. Decompose $M_{2}$ as

$$
\begin{aligned}
M_{2}= & \frac{1}{n h f(x)} \sum_{i=1}^{n}\left\{K_{h}\left(x-X_{i}\right) \sigma^{2}\left(X_{i}\right) \xi_{i}-\mathrm{E}\left[K_{h}\left(x-X_{i}\right) \sigma^{2}\left(X_{i}\right) \xi_{i} \mid \mathcal{G}_{i-1}\right]\right\} \\
& +\frac{1}{n h f(x)} \mathrm{E}\left[\sigma^{2}\left(X_{1}\right) K_{h}\left(x-X_{1}\right)\right] \sum_{i=1}^{n} \mathrm{E}\left[\xi_{i} \mid \mathcal{H}_{i-1}\right]=: M_{21}+M_{22} .
\end{aligned}
$$

Using the martingale CLT once again, we conclude that

$$
\sqrt{n h f(x)} M_{21} \stackrel{\mathrm{d}}{\rightarrow} \mathcal{N}\left(0, \sigma^{4}(x) \mathrm{E}\left[\xi_{1}^{2}\right] \int K^{2}\right) .
$$

Moreover, $\mathrm{E}\left[M_{22}\right]=0$ and with $d_{n}$ defined in (2.9),

$$
M_{22}=\sigma^{2}(x) \frac{d_{n}}{n} d_{n}^{-1} \sum_{i=1}^{n} \mathrm{E}\left[\xi_{i} \mid \mathcal{H}_{i-1}\right]+O(h) \frac{d_{n}}{n} d_{n}^{-1} \sum_{i=1}^{n} \mathrm{E}\left[\xi_{i} \mid \mathcal{H}_{i-1}\right] .
$$


If $\alpha_{\varepsilon}<1 / 2$ we use (2.9) and (2.11) to get

$$
n^{\alpha_{\varepsilon}} M_{22} \stackrel{\mathrm{d}}{\rightarrow} C_{2} \sigma^{2}(x) Z_{2}(1) .
$$

Comparing (5.12) with (5.13) we note that either $M_{21}$ or $M_{22}$ dominates, according to the respective assumptions on $h$, as stated in the Proposition 3.2. If $h n^{\left(1-2 \alpha_{\varepsilon}\right)} \rightarrow 0$ then $M_{21}$ is of the higher order than $M_{22}$ and the limiting behaviour of $\left\{\hat{\sigma}_{\text {oracle }}^{2}(x)-\sigma^{2}(x)\right\}$ is determined by $M_{21}$. Otherwise, if $h n^{\left(1-2 \alpha_{\varepsilon}\right)} \rightarrow \infty$, then $M_{22}$ determines the limiting behaviour of the oracle estimator. On the other hand, if $\alpha_{\varepsilon}>1 / 2, M_{22}=O_{P}\left(n^{-1 / 2}\right)$ (see (2.10)), so that $M_{22}$ is negligible w.r.t. $M_{21}$.

Under (E2) and (P2) we start once again with the following martingale decomposition (recall $M_{21}$ from (5.11)):

$$
\begin{aligned}
& M_{21}+\frac{1}{n h f(x)} \sum_{i=1}^{n} \mathrm{E}\left[K_{h}\left(x-X_{i}\right) \sigma^{2}\left(X_{i}\right) \xi_{i} \mid \mathcal{G}_{i-1}\right] \\
& =M_{21}+\frac{1}{n h f(x)} \sum_{i=1}^{n} \mathrm{E}\left[\xi_{i} \mid \mathcal{H}_{i-1}\right] \int K_{h}\left(x-z \varphi\left(U_{i}\right)\right) \sigma^{2}\left(z \varphi\left(U_{i}\right)\right) f_{Z}(z) d v .
\end{aligned}
$$

Again, a function $\left.\Lambda(\cdot ; x) \rightarrow \int K_{h}(x-z \varphi(\cdot)) \sigma^{2}(z \varphi(\cdot))\right) f_{Z}(z) d z$ is in $L^{2}(\phi)$. Indeed, its $L^{2}(\phi)$ norm can be bounded by $\sup _{x}\left|K^{2}(x)\right| \mathrm{E}\left[\sigma^{2}(X)\right]$, which is finite on account of (D2). Furthermore, let us assume that its centered version has Hermite rank $q^{\prime} \geq 1$. Let $\tilde{J}_{m, h}^{*}(x), m \geq q^{\prime}$, be the corresponding Hermite coefficients, i.e.

$$
\tilde{J}_{m, h}^{*}(x)=\mathrm{E}\left[H_{m}\left(U_{1}\right) \Lambda\left(U_{1} ; x\right)\right],
$$

where $U_{1}$ is standard normal. Put $\Psi\left(\varepsilon_{i}\right):=\mathrm{E}\left[\xi_{i} \mid \mathcal{H}_{i-1}\right]$. With this notation we can re-write the second part in (5.14) as

$$
\begin{aligned}
M_{23}:= & \frac{1}{n h f(x)} \sum_{i=1}^{n} \mathrm{E}\left[\xi_{i} \mid \mathcal{H}_{i-1}\right] \Lambda\left(U_{i} ; x\right) \\
= & \int \frac{1}{n h} \mathrm{E}\left[K_{h}\left(x-z \varphi\left(U_{1}\right)\right) \sigma^{2}\left(v \varphi\left(U_{1}\right)\right)\right] f_{Z}(z) d z \frac{1}{f(x)} \sum_{i=1}^{n} \Psi\left(\varepsilon_{i}\right) \\
& +\frac{1}{n h f(x)} \sum_{i=1}^{n} \Psi\left(\varepsilon_{i}\right) \sum_{m=q^{\prime}}^{\infty} \tilde{J}_{m, h}^{*}(x) \frac{H_{m}\left(U_{i}\right)}{m !} .
\end{aligned}
$$

The first integral in the expression for $J_{23}$ is evaluated as

$$
\begin{aligned}
& \iint K_{h}(x-z y) \sigma^{2}(z y) f_{Z}(z) f_{\varphi(U)}(y) d y d z \\
& \quad \sim h \sigma^{2}(x) \mathrm{E}\left[f_{\varphi(U)}\left(\frac{x}{Z_{1}}\right) \frac{1}{Z_{1}}\right]=h \sigma^{2}(x) J_{0}^{*}(x) .
\end{aligned}
$$


Therefore, by (2.11), the first part in $M_{23}$ behaves like

$$
\frac{\sigma^{2}(x) J_{0}^{*}(x)}{n f(x)} \sum_{i=1}^{n} \mathrm{E}\left[\xi_{i} \mid \mathcal{H}_{i-1}\right]=O_{P}\left(n^{-\alpha_{\varepsilon}} \vee n^{-1 / 2}\right) .
$$

Without loss of generality we may assume that $\mathrm{E}\left[U_{i} U_{j}\right]<1$ for all $i \neq j$. With help of Mehler's formula and (2.8), the variance of $M_{23}$ can be written as

$$
\begin{aligned}
& \operatorname{Var}\left(\frac{1}{n h f(x)} \sum_{i=1}^{n} \Psi\left(\varepsilon_{i}\right) \Lambda\left(U_{i} ; x\right)\right) \\
& =\frac{1}{n^{2} h^{2} f^{2}(x)} \sum_{i, j=1}^{n} \mathrm{E}\left[\Psi\left(\varepsilon_{i}\right) \Psi\left(\varepsilon_{j}\right)\right] \mathrm{E}\left[\Lambda\left(U_{i} ; x\right) \Lambda\left(U_{j} ; x\right)\right] \\
& =\frac{1}{n^{2} h^{2} f^{2}(x)} \sum_{i, j=1}^{n} \mathrm{E}\left[\Psi\left(\varepsilon_{i}\right) \Psi\left(\varepsilon_{j}\right)\right] \sum_{m=q^{\prime}}^{\infty} \frac{\tilde{J}_{m, h}^{* 2}(x)}{m !} \mathrm{E}^{m}\left[U_{i} U_{j}\right] \\
& \leq\|\Lambda(\cdot ; x)\|_{L^{2}(\phi)}^{2} \frac{1}{n^{2} h^{2} f^{2}(x)} \sum_{i, j=1}^{n} \mathrm{E}\left[\Psi\left(\varepsilon_{i}\right) \Psi\left(\varepsilon_{j}\right)\right]\left|\mathrm{E}^{q^{\prime}}\left[U_{i} U_{j}\right]\right| \\
& \quad=\frac{1}{h^{2} f^{2}(x)}\|\Lambda(\cdot ; x)\|_{L^{2}(\phi)}^{2} O\left(n^{-1} \vee n^{-\left(2 \alpha_{\varepsilon}+q \alpha_{U}\right)}\right) .
\end{aligned}
$$

Applying (5.32) below, $\tilde{J}_{m, h}^{* 2}(x) \sim h^{2} \sigma^{4}(x) J_{m}^{* 2}(x)$, as $h \rightarrow 0$. Therefore,

$$
\|\Lambda(\cdot ; x)\|_{L^{2}(\phi)}^{2}=O\left(h^{2} \sigma^{4}(x)\right)\left\|\frac{1}{\varphi(\cdot)} f_{Z}\left(\frac{x}{\varphi(\cdot)}\right)\right\|_{L^{2}(\phi)}^{2} .
$$

Consequently, we conclude that $M_{23}=O_{P}\left(n^{-1 / 2}\right)$ if $\alpha_{\varepsilon}>1 / 2$. Otherwise, if $\alpha_{\varepsilon}<1 / 2$ then the limiting behaviour of $n^{\alpha_{\varepsilon}} M_{23}$ is the same as the term in $(5.15)$, i.e.

$$
n^{\alpha_{\varepsilon}} \frac{\sigma^{2}(x) J_{0}^{*}(x)}{n f(x)} \sum_{i=1}^{n} \mathrm{E}\left[\xi_{i} \mid \mathcal{H}_{i-1}\right] \stackrel{\mathrm{d}}{\rightarrow} C_{2} \sigma^{2}(x) \frac{J_{0}^{*}(x)}{f(x)} Z_{2}(1) .
$$

Now, again, asymptotic behaviour of $M_{2}$ in (5.8) is obtained by comparing $M_{21}$ in (5.12) with (5.16) under the respective assumptions on $h$.

Remark 5.2. If $f$ is estimated by $\hat{f}_{h}$, then $M_{1}$ becomes basically the stochastic bias term. Consider for a moment the case of (P2) with $X_{i}=U_{i}$. Let us assume that the density $f$ is known. Then,

$$
\tilde{M}_{1}:=\left(\frac{1}{n h f(x)} \sum_{i=1}^{n} \sigma^{2}\left(X_{i}\right) K_{h}\left(x-X_{i}\right)-\sigma^{2}(x)\right)
$$

can be decomposed in the analogous way as the kernel density estimator in (5.1). Since now $q=1$, we conclude that under (D3) the proper normalization for $\tilde{M}_{1}$ is either $\sqrt{n h f(x)}$ if $h n^{\left(1-\alpha_{X}\right)} \rightarrow 0$, or $n^{\alpha_{X} / 2}$ if $h n^{\left(1-\alpha_{X}\right)} \rightarrow \infty$. This explains difference between our results and those in Guo and Koul (2008). A similar effect was also mentioned in Mielniczuk and $\mathrm{Wu}$ (2004). 


\subsection{Proof of Theorems 3.5 and 3.6}

Recall the decomposition (5.3). Asymptotic behaviour of $M_{1}$ and $M_{2}$ ((5.5) and (5.6), respectively) was proven in Section 3.1. Thus, in order to prove Theorem 3.5 (assuming that (E2) and either (P1) or (P2) holds) and Theorem 3.6 (assuming that (E2)+(P2) holds) it is enough to study $M_{3}$ and $M_{4}$. We will show that in the first case $\sqrt{n h}\left(M_{3}+M_{4}\right)=o_{P}(1)$, whereas in the second case (5.9) and (5.10) hold. Specifically, the proof of theorems will be finished given that we validate the following lemmas.

Lemma 5.3. Under the conditions of Theorem 3.5 we have $\sqrt{n h} M_{3}=o_{P}(1)$. Under the conditions of Theorem 3.6, (5.9) holds.

Lemma 5.4. Under the conditions of Theorem 3.5 we have $\sqrt{n h} M_{4}=o_{P}(1)$. Under the conditions of Theorem 3.6, (5.10) holds.

Least squares estimation of regression parameters leads to the following expressions:

$$
\hat{\beta}_{1}-\beta_{1}=\frac{1}{S_{n}}\left(\frac{1}{n} \sum_{j=1}^{n} X_{j} \sigma\left(X_{j}\right) \varepsilon_{j}-\bar{X} \bar{V}\right), \hat{\beta}_{0}-\beta_{0}=\bar{V}-\bar{X}\left(\hat{\beta}_{1}-\beta_{1}\right),(5
$$

where $\bar{X}$ and $\bar{V}$ are sample means based on $X_{1}, \ldots, X_{n}$ and $\sigma\left(X_{1}\right) \varepsilon_{1}, \ldots, \sigma\left(X_{n}\right) \varepsilon_{n}$, respectively, and $S_{n}=\frac{1}{n} \sum_{j=1}^{n}\left(X_{j}-\bar{X}\right)^{2}$.

Proof of Lemma 5.3. Again, we may replace $\hat{f}_{h}$ with $f$ in the expression for $M_{3}$. Write

$$
\begin{aligned}
M_{3}= & \left(\hat{\beta}_{0}-\beta_{0}\right) \frac{2}{n h f(x)} \sum_{i=1}^{n} K_{h}\left(x-X_{i}\right) \sigma\left(X_{i}\right) \varepsilon_{i} \\
& +\left(\hat{\beta}_{1}-\beta_{1}\right) \frac{2}{n h f(x)} \sum_{i=1}^{n} K_{h}\left(x-X_{i}\right) X_{i} \sigma\left(X_{i}\right) \varepsilon_{i} \\
=: & L_{3}+R_{3}=:\left(\hat{\beta}_{0}-\beta_{0}\right) \tilde{L}_{3}+\left(\hat{\beta}_{1}-\beta_{1}\right) \tilde{R}_{3} .
\end{aligned}
$$

Under (E1) (regardless whether (P1) or (P2) holds) both $\tilde{L}_{3}$ and $\tilde{R}_{3}$ are martingales so that both are $O_{P}\left((n h)^{-1 / 2}\right)$. Indeed, for example for $\tilde{L}_{3}$ we have

$$
\begin{aligned}
\operatorname{Var}\left(\tilde{L}_{3}\right) & =\frac{1}{n^{2} h^{2} f^{2}(x)} \sum_{i=1}^{n} \operatorname{Var}\left(K_{h}\left(x-X_{i}\right) \sigma\left(X_{i}\right) \varepsilon_{i}\right) \\
& \leq \frac{1}{n h^{2} f^{2}(x)} \mathrm{E}\left[\varepsilon^{2}\right] \mathrm{E}\left[K_{h}^{2}\left(x-X_{1}\right) \sigma\left(X_{1}\right)\right]=O\left(\frac{1}{n h}\right) .
\end{aligned}
$$

Since $\hat{\beta}_{0}$ and $\hat{\beta}_{1}$ are consistent, we conclude that $\sqrt{n h} M_{3}=o_{P}(1)$.

For the case of $(\mathrm{E} 2)+(\mathrm{P} 1)$, let us define the quantity

$$
\tilde{M}_{3}:=\frac{2}{n^{2} h f(x)} \sum_{i=1}^{n} \sum_{j=1}^{n} K_{h}\left(x-X_{i}\right) \sigma\left(X_{i}\right) \sigma\left(X_{j}\right)\left(X_{i}-\mu\right)\left(X_{j}-\mu\right) \varepsilon_{i} \varepsilon_{j} .
$$


We may decompose

$$
M_{3}=\frac{1}{S_{n}} \tilde{M}_{3}+\bar{V} \tilde{L}_{3}-\frac{1}{S_{n}}(\bar{X}-\mu) \bar{V}\left(\tilde{R}_{3}-\mu \tilde{L}_{3}\right)-(\bar{X}-\mu)\left(\hat{\beta}_{1}-\beta_{1}\right) \tilde{L}_{3} .
$$

On account of Slutsky's lemma, we may ignore $S_{n}$ when studying asymptotic of $M_{3}$. Under (E2) and (P1), it will be shown below that $\sqrt{n h} \tilde{M}_{3}=o_{P}(1)$ if $\mathrm{E}\left[\sigma\left(X_{1}\right)\left(X_{1}-\mu\right)\right]=0$ and

$$
n^{\alpha_{\varepsilon}} \tilde{M}_{3} \stackrel{\mathrm{d}}{\rightarrow}\left(2 C_{1}^{2} \mathrm{E}\left[\sigma\left(X_{1}\right)\left(X_{1}-\mu\right)\right] \sigma(x)(x-\mu)\right) \times \chi^{2}(1)
$$

otherwise. For the second term in (5.18) we will prove

$$
n^{\alpha_{\varepsilon}} \bar{V} \tilde{L}_{3} \stackrel{\mathrm{d}}{\rightarrow}\left(2 C_{1}^{2} \sigma(x) \mathrm{E}\left[\sigma\left(X_{1}\right)\right]\right) \times \chi^{2}(1) .
$$

Furthermore, the third term is $O_{p}\left(n^{-1 / 2}\right)=o_{P}\left((n h)^{-1 / 2}\right)$, which is obvious since $\bar{X}-\mu=O_{P}\left(n^{-1 / 2}\right)$ and $\left(\tilde{R}_{3}-\mu \tilde{L}_{3}\right)=O_{P}(1)$ (the latter may be concluded by showing that $\mathrm{E}\left[\left|\tilde{R}_{3}\right|\right]<\infty$ and $\mathrm{E}\left[\left|\tilde{L}_{3}\right|\right]<\infty$. Indeed, for $\left|\tilde{R}_{3}\right|$ we have

$$
\left.\mathrm{E}\left[\left|\tilde{R}_{3}\right|\right] \leq \frac{2}{n h f(x)} n \mathrm{E}\left[\left|K_{h}\left(x-X_{i}\right) X_{i} \sigma\left(X_{1}\right)\right|\right] \mathrm{E}\left[\left|\varepsilon_{1}\right|\right]=O(1) .\right)
$$

Also,

$$
n^{\alpha_{\varepsilon} / 2} \bar{V} \stackrel{\mathrm{d}}{\rightarrow} \mathcal{N}\left(0, C_{1}^{2} \mathrm{E}^{2}\left[\sigma\left(X_{1}\right)\right]\right)
$$

so that $\bar{V}=o_{P}(1)$. Likewise, the fourth term is $o_{P}\left(n^{-1 / 2}\right)=o_{P}\left((n h)^{-1 / 2}\right)$. Equations (5.19), (5.20) and the fact that the convergence in (5.19) and (5.20) is joint (this follows from the proof below) imply (5.9).

Thus, let us deal with the contributing terms $\tilde{M}_{3}$ and $\bar{V} \tilde{L}_{3}$. Under (E2) and (P1), define

$$
V_{h}\left(x, X_{i}, X_{j}\right):=K_{h}\left(x-X_{i}\right) \sigma\left(X_{i}\right) \sigma\left(X_{j}\right)\left(X_{i}-\mu\right)\left(X_{j}-\mu\right) .
$$

Let $\bar{V}_{h}\left(x, X_{i}, X_{j}\right):=V_{h}\left(x, X_{i}, X_{j}\right)-\mathrm{E}\left[V_{h}\left(x, X_{i}, X_{j}\right)\right]$. Consider the following decomposition

$$
\begin{aligned}
\tilde{M}_{3}= & \frac{2}{n^{2} h f(x)} \sum_{i=1}^{n} \varepsilon_{i}^{2} V_{h}\left(x, X_{i}, X_{i}\right)+\frac{2}{n^{2} h f(x)} \sum_{\substack{i, j=1 \\
i \neq j}}^{n} \varepsilon_{i} \varepsilon_{j} \mathrm{E}\left[V_{h}\left(x, X_{i}, X_{j}\right)\right] \\
& +\frac{2}{n^{2} h f(x)} \sum_{\substack{i, j=1 \\
i \neq j}}^{n} \varepsilon_{i} \varepsilon_{j} \bar{V}_{h}\left(x, X_{i}, X_{j}\right)=: M_{31}+M_{32}+M_{33} .
\end{aligned}
$$

We note that $\mathrm{E}\left[V_{h}\left(x, X_{1}, X_{1}\right)\right]$ is proportional to $h$. Indeed, using the first order Taylor's expansion,

$\mathrm{E}\left[V_{h}\left(x, X_{1}, X_{1}\right)\right]=\int K_{h}(x-s) \sigma^{2}(s)(s-\mu)^{2} f_{X}(s) d s \sim h \sigma^{2}(x)(x-\mu)^{2} f_{X}(x)$, 
where $f_{X}$ is the density of $X_{1}$. Therefore, the first term in $\tilde{M}_{3}$ is of order $O_{P}(1 / n)$, so that $\sqrt{n h} M_{31}=o_{P}(1)$. In what follows, we will show that $\sqrt{n h} J_{33}=o_{P}(1)$ and the only part which may contribute is $M_{32}$.

The second part, $M_{32}$, is written as (recall the definition of $b_{n}^{2}$ in (2.5))

$$
\frac{2 \mathrm{E}\left[V_{h}\left(x, X_{1}, X_{2}\right)\right] b_{n}^{2}}{n^{2} h f(x)}\left\{\left(b_{n}^{-1} \sum_{j=1}^{n} \varepsilon_{j}\right)^{2}-b_{n}^{-2} \sum_{j=1}^{n} \varepsilon_{j}^{2}\right\} .
$$

The second part in the brackets is negligible. Furthermore, note that

$$
\begin{aligned}
\mathrm{E} & {\left[V_{h}\left(x, X_{1}, X_{2}\right)\right]=\mathrm{E}\left[K_{h}\left(x-X_{i}\right) \sigma\left(X_{i}\right) \sigma\left(X_{j}\right)\left(X_{i}-\mu\right)\left(X_{j}-\mu\right)\right] } \\
& =\int K_{h}(x-s) \sigma(s) \sigma(t)(s-\mu)(t-\mu) f(s) f(t) d s d t \\
& =h \int K(v) \sigma(x-v h) \sigma(t)(x-v h-\mu)(t-\mu) f(x-v h) f(t) d v d t \\
& \sim h \sigma(x)(x-\mu) f(x) \mathrm{E}\left[\sigma\left(X_{1}\right)\left(X_{1}-\mu\right)\right] .
\end{aligned}
$$

Using (5.24) we obtain (5.19). If $\mathrm{E}\left[\sigma\left(X_{1}\right)\left(X_{1}-\mu\right)\right]=0$, then $\mathrm{E}\left[V_{h}\left(x, X_{1}, X_{2}\right)\right]=$ 0 and $M_{32} \equiv 0$.

Next, we establish a bound on $M_{33}$. We have

$$
\begin{aligned}
& \operatorname{Var}\left(\sum_{\substack{j, i=1 \\
j \neq i}}^{n} \varepsilon_{i} \varepsilon_{j} \bar{V}_{h}\left(x, X_{i}, X_{j}\right)\right) \leq \sum_{\substack{i, j=1 \\
j \neq i}}^{n} \mathrm{E}\left[\varepsilon_{i}^{2} \varepsilon_{j}^{2}\right] \mathrm{E}\left[\bar{V}_{h}^{2}\left(x, X_{i}, X_{j}\right)\right] \\
& \quad+\sum_{i=1}^{n} \sum_{\substack{j, j^{\prime}=1 \\
j^{\prime} \neq j, j \neq i, j^{\prime} \neq i}}^{n} \mathrm{E}\left[\varepsilon_{i}^{2} \varepsilon_{j} \varepsilon_{j^{\prime}}\right] \mathrm{E}\left[\bar{V}_{h}\left(x, X_{i}, X_{j}\right) \bar{V}_{h}\left(x, X_{i}, X_{j^{\prime}}\right)\right] \\
& \quad+\sum_{j=1}^{n} \sum_{\substack{i, i^{\prime}=1 \\
i^{\prime} \neq i, i \neq j, i^{\prime} \neq j}}^{n} \mathrm{E}\left[\varepsilon_{j}^{2} \varepsilon_{i} \varepsilon_{i^{\prime}}\right] \mathrm{E}\left[\bar{V}_{h}\left(x, X_{i}, X_{j}\right) \bar{V}_{h}\left(x, X_{i^{\prime}}, X_{j}\right)\right] \\
& \quad+\sum_{i=1}^{n} \sum_{\substack{j, j^{\prime}=1 \\
i \neq j, j \neq j^{\prime}}}^{n} \mathrm{E}\left[\varepsilon_{i} \varepsilon_{j}^{2} \varepsilon_{j^{\prime}}\right] \mathrm{E}\left[\bar{V}_{h}\left(x, X_{i}, X_{j}\right) \bar{V}_{h}\left(x, X_{j}, X_{j^{\prime}}\right)\right] \\
& \quad+\sum_{\substack{i, i^{\prime}=1 \\
i \neq j, i \neq i^{\prime}}}^{n} \sum_{j=1}^{n} \mathrm{E}\left[\varepsilon_{i}^{2} \varepsilon_{j} \varepsilon_{i^{\prime}}\right] \mathrm{E}\left[\bar{V}_{h}\left(x, X_{i}, X_{j}\right) \bar{V}_{h}\left(x, X_{i^{\prime}}, X_{i}\right)\right] \\
& \quad+\sum_{\substack{i, i^{\prime}, j, j^{\prime} \\
i \neq i^{\prime} \neq j \neq j^{\prime}}} \mathrm{E}\left[\varepsilon_{i} \varepsilon_{i^{\prime}} \varepsilon_{j} \varepsilon_{j^{\prime}}\right] \mathrm{E}\left[\bar{V}_{h}\left(x, X_{i}, X_{j}\right) \bar{V}_{h}\left(x, X_{i^{\prime}}, X_{j^{\prime}}\right)\right] .
\end{aligned}
$$

The last part is equal to 0 , since for different indices $\bar{V}_{h}\left(x, X_{i}, X_{j}\right), \bar{V}_{h}\left(x, X_{i^{\prime}}, X_{j^{\prime}}\right)$ are independent (and centered). Next, straightforward computation shows that

$$
\mathrm{E}\left[V_{h}^{2}\left(x, X_{i}, X_{j}\right)\right]=O(h),
$$




$$
\mathrm{E}\left[V_{h}\left(x, X_{i}, X_{j}\right) V_{h}\left(x, X_{i^{\prime}}, X_{j^{\prime}}\right)\right]=O\left(h^{2}\right), \quad i \neq i^{\prime},
$$

and

$$
\mathrm{E}\left[V_{h}\left(x, X_{i}, X_{j}\right) V_{h}\left(x, X_{i}, X_{j^{\prime}}\right)\right]=O(h), \quad i \neq j .
$$

Using the covariance bounds on the error sequence, we obtain that the variance above is of the order $O\left(h n^{3-\alpha_{\varepsilon}}+h n^{2}\right)$. Consequently,

$$
\operatorname{Var}\left(M_{33}\right)=O\left(\frac{n^{2} h}{n^{4} h^{2}}+\frac{h n^{3-\alpha_{\varepsilon}}}{n^{4} h^{2}}\right)=O\left(\frac{1}{n^{2} h}+\frac{1}{h n^{1+\alpha_{\varepsilon}}}\right),
$$

and so $\sqrt{n h} M_{33}=o_{P}(1)$.

The proof of (5.20) follows along the same lines as for (5.19). Furthermore, we note that the convergence in (5.19) follows from the asymptotic expansion of $\tilde{M}_{3}$ with the dominating term as in (5.23). The same applies to the convergence (5.20) - up to a deterministic constant, the term (5.23) appears in the asymptotic expansion for $\bar{V} \tilde{L}_{3}$. Therefore, the convergence in (5.19) and (5.20) is joint, so that we may conclude (5.9). This finishes the proof of Lemma 5.3.

Remark 5.5. Note that the second term in the decomposition (5.18) is the effect of the estimation of the intercept $\beta_{0}$ and contributes with rate $n^{-\alpha_{\varepsilon}}$. If the model (1.1) is considered without intercept $\beta_{0}$, then this term does not appear, so that $n^{-\alpha_{\varepsilon}}$ contribution appears only when $\mathrm{E}\left[\sigma\left(X_{1}\right)\left(X_{1}-\mu\right)\right] \neq 0$.

To prove Lemma 5.4, we establish limiting behaviour if $\hat{\beta}_{1}$ in a particular case.

Lemma 5.6. Assume (E2) and (P1). If $\mathrm{E}\left[\sigma\left(X_{1}\right)\left(X_{1}-\mu\right)\right] \neq 0$ holds, then

$$
n^{\alpha_{\varepsilon} / 2}\left(\hat{\beta}_{1}-\beta_{1}\right) \stackrel{d}{\rightarrow} \mathcal{N}\left(0, \omega^{2}\right) .
$$

Proof of Lemma 5.6. Recall from (5.17) that

$$
\hat{\beta}_{1}-\beta_{1}=\frac{1}{S_{n}}\left(\frac{1}{n} \sum_{j=1}^{n} X_{j} \sigma\left(X_{j}\right) \varepsilon_{j}-\bar{X} \bar{V}\right),
$$

where $S_{n}^{2}$ is the sample variance associated with $X_{1}, \ldots, X_{n}$. Since $\operatorname{Var}\left[X_{1}^{2}\right]=1$, $S_{n} \stackrel{\mathrm{P}}{\rightarrow} 1$. Thus, via Slutsky's lemma, in order to show convergence of $\hat{\beta}_{1}-\beta_{1}$, it suffices to study

$$
I_{1}-I_{2}:=\frac{1}{n} \sum_{j=1}^{n} X_{j} \sigma\left(X_{j}\right) \varepsilon_{j}-\bar{X} \bar{V} .
$$

Consider the sum $\frac{1}{n} \sum_{j=1}^{n} \nu\left(X_{j}\right) \varepsilon_{j}$, where $\nu(\cdot)$ is a deterministic function. Decompose this sum into:

$$
\frac{1}{n} \sum_{i=1}^{n}\left(\nu\left(X_{i}\right) \varepsilon_{i}-\mathrm{E}\left[\nu\left(X_{i}\right) \varepsilon_{i} \mid \mathcal{G}_{i-1}\right]\right)+\mathrm{E}\left[\nu\left(X_{1}\right)\right] \frac{1}{n} \sum_{i=1}^{n} \mathrm{E}\left[\varepsilon_{i} \mid \mathcal{H}_{i-1}\right]
$$


The first part is a martingale. If $\mathrm{E}\left[\nu\left(X_{1}\right)\right] \neq 0$, the second part is of order $O_{P}\left(n^{-\alpha_{\varepsilon} / 2}\right)$, and dominates the martingale part. In this case its convergence follows from (2.5) and (2.7). Otherwise, if $\mathrm{E}\left[\nu\left(X_{1}\right)\right]=0$, the second part disappears and the sum is $O_{P}\left(n^{-1 / 2}\right)$.

Such decomposition is applied to $I_{1}-I_{2}$ :

$$
\begin{aligned}
I_{1}-I_{2} & =\frac{1}{n} \sum_{j=1}^{n} X_{j} \sigma\left(X_{j}\right) \varepsilon_{j}-I_{2} \\
= & \frac{1}{n} \sum_{j=1}^{n}\left(X_{j} \sigma\left(X_{j}\right) \varepsilon_{j}-\mathrm{E}\left[X_{j} \sigma\left(X_{j}\right) \varepsilon_{j} \mid \mathcal{G}_{j-1}\right]\right) \\
& +\mathrm{E}\left[X_{1} \sigma\left(X_{1}\right)\right] \frac{1}{n} \sum_{j=1}^{n} \mathrm{E}\left[\varepsilon_{j} \mid \mathcal{H}_{j-1}\right]-I_{2}=: A_{1}+A_{2}-I_{2} .
\end{aligned}
$$

We have $\sqrt{n} A_{1} \stackrel{\mathrm{d}}{\rightarrow} \mathcal{N}\left(0, \sigma_{0}^{2}\right)$. Convergence for $A_{2}$ follows from (2.7). The term $I_{2}$ is $O_{P}\left(n^{-\left(1+\alpha_{\varepsilon}\right) / 2}\right)$ (see $\left.(2.5)\right)$ so that it is negligible.

Proof of Lemma 5.4: Using (5.17), we decompose $M_{4}$ as follows:

$$
\begin{aligned}
M_{4} & =\frac{1}{n h f(x)}(\bar{V})^{2} \sum_{i=1}^{n} K_{h}\left(x-X_{i}\right)+\frac{1}{n h f(x)}\left(\hat{\beta}_{1}-\beta_{1}\right)^{2} \sum_{i=1}^{n}\left(X_{i}-\bar{X}\right)^{2} K_{h}\left(x-X_{i}\right) \\
& +\frac{2}{n h f(x)} \bar{V}\left(\hat{\beta}_{1}-\beta_{1}\right) \sum_{i=1}^{n}\left(X_{i}-\bar{X}\right) K_{h}\left(x-X_{i}\right)=: B_{1}+B_{2}+B_{3} .
\end{aligned}
$$

Under (E1), we have clearly $M_{4}=O_{P}\left(n^{-1}\right)$, which clearly implies $M_{4}=$ $O_{P}\left((n h)^{-1 / 2}\right)$.

Under (E2) and (P1), we obtain via (5.21) that

$$
n^{\alpha_{\varepsilon}} B_{1} \stackrel{\mathrm{d}}{\rightarrow} C_{1}^{2} \mathrm{E}^{2}\left[\sigma\left(X_{1}\right)\right] \chi^{2}(1) .
$$

Note that $B_{1}$ has a structure $(\bar{V})^{2} \times V_{n}$, where $V_{n}=\frac{1}{n h f(x)} \sum_{i=1}^{n} K_{h}\left(x-X_{i}\right)$. We decompose the term as $(\bar{V})^{2} \mathrm{E}\left[V_{n}\right]+(\bar{V})^{2}\left(V_{n}-\mathrm{E}\left[V_{n}\right]\right)$. Then the second term is $o_{P}\left((\bar{V})^{2}\right)$ and $\mathrm{E}\left[V_{n}\right] \sim 1$. Likewise, from Lemma 5.6,

$$
n^{\alpha_{\varepsilon}} B_{2} \stackrel{\mathrm{d}}{\rightarrow}(x-\mu)^{2} \omega^{2} \chi^{2}(1)
$$

if $\mathrm{E}\left[\sigma\left(X_{1}\right)\left(X_{1}-\mu\right)\right] \neq 0$ and $B_{2}=O_{P}\left(n^{-1}\right)$ otherwise. For the term $B_{3}$ we have clearly that (recall $(\mathrm{P} 1))(n h)^{-1} \sum_{i=1}^{n}\left(X_{i}-\bar{X}\right) K_{h}\left(x-X_{i}\right)=O_{P}\left((n h)^{-1 / 2}\right)$, so that $B_{3}=o_{P}\left((n h)^{-1 / 2}\right)$. Furthermore, as in case of $M_{3}$, the convergence in (5.30) and (5.20) is concluded from the asymptotic expansions, so that it is joint and (5.10) may be concluded. Furthermore, the convergence in (5.9) and (5.10) is joint. 


\section{Appendix}

\section{Assumption (J2)}

Let $\zeta$ be a real function. Consider a function

$$
(\cdot ; x, h, \zeta) \rightarrow \int K_{h}(x-z \varphi(\cdot)) \zeta(z \varphi(\cdot)) f_{Z}(z) d z
$$

and its corresponding Hermite coefficient $J_{m, h}^{*}(x ; \zeta)$. If $\zeta$ is in $\mathcal{C}^{2}(\mathcal{I})$, then one can evaluate

$$
J_{m, h}^{*}(x ; \zeta) \sim h \zeta(x) J_{m}^{*}(x):=h \zeta(x) \mathrm{E}\left[J_{m}^{\prime}\left(\frac{x}{Z_{1}}\right) \frac{1}{Z_{1}}\right] .
$$

Indeed, to see this assume for simplicity that $\varphi$ is invertible. Then changing variables $u \rightarrow s=(x-z \varphi(u)) / h$ and applying Taylor expansion,

$$
\begin{aligned}
& J_{m, h}^{*}(x)=\iint K_{h}(x-z \varphi(u)) \zeta(z \varphi(u)) f_{Z}(z) \phi(u) H_{m}(u) d z d u \\
& =h \iint \phi\left(\varphi^{-1}\left(\frac{x-s h}{z}\right)\right) H_{m}\left(\varphi^{-1}\left(\frac{x-s h}{z}\right)\right) \frac{1}{z} \frac{K(s) \zeta(x-s h) f_{Z}(z)}{\varphi^{\prime}\left(\varphi^{-1}\left(\frac{x-s h}{z}\right)\right)} d z d s \\
& \quad \sim h \zeta(x) \int \phi\left(\varphi^{-1}\left(\frac{x}{z}\right)\right) H_{m}\left(\varphi^{-1}\left(\frac{x}{z}\right)\right) \frac{1}{\varphi^{\prime}\left(\varphi^{-1}\left(\frac{x}{z}\right)\right)} \frac{1}{z} f_{Z}(z) d z \\
& =h \zeta(x) \int J_{m}^{\prime}\left(\frac{x}{z}\right) \frac{1}{z} f_{Z}(z) d z=h \zeta(x) \mathrm{E}\left[J_{m}^{\prime}\left(\frac{x}{Z_{1}}\right) \frac{1}{Z_{1}}\right] .
\end{aligned}
$$

Substituting further $u=\varphi^{-1}(x / z)$ we evaluate integral in (5.33) as

$$
\int \phi(u) H_{m}(u) \frac{1}{\varphi(u)} f_{Z}\left(\frac{x}{\varphi(u)}\right) d u .
$$

The latter expression can be recognized as $m$ th Hermite coefficient of function $(\cdot ; x) \rightarrow \frac{1}{\varphi(\cdot)} f_{Z}\left(\frac{x}{\varphi(\cdot)}\right)$. Therefore, assumption (J2) guarantees that coefficients $J_{m}^{*}(x)$ are well defined for each $m$. We can also see that if $f_{Z}$ is degenerated (i.e. it has mass one at $x=1$, say), then (J2) reduces to (J1).

\section{Martingale CLT}

Let us recall from Hall and Heyde (1980) the following martingale central limit theorem.

Lemma 5.7. Assume that $\left(R_{i}, \mathcal{G}_{i}\right), i \geq 1$, is a martingale difference. Define $\bar{R}_{i}=R_{i}-\mathrm{E}\left[R_{i} \mid \mathcal{G}_{i-1}\right]$. If

$$
\sum_{i=1}^{n} \mathrm{E}\left[\bar{R}_{i}^{2} 1_{\left\{\left|\bar{R}_{i}\right|>\delta\right\}}\right] \rightarrow 0 \quad \text { for each } \delta>0
$$


and

$$
\sum_{i=1}^{n} \mathrm{E}\left[\bar{R}_{i}^{2} \mid \mathcal{G}_{i-1}\right] \stackrel{P}{\rightarrow} 1
$$

then

$$
\sum_{i=1}^{n} R_{i} \stackrel{d}{\rightarrow} N(0,1)
$$

To obtain the limit theorem for the term $J_{21}$ in (5.11) we consider

$$
R_{i}=(n h)^{-1 / 2} \sigma^{2}\left(X_{i}\right) K_{h}\left(x-X_{i}\right) \xi_{i} / \sqrt{f(x)} .
$$

The proof is exactly the same as in Wu and Mielniczuk (2002, Lemma 2) and therefore it is omitted.

\section{Acknowledgement}

This work was started while the second author visited the Department of Mathematics and Statistics at the University of Ottawa. The visit was partially supported by the Robert Bosch Stiftung. The work of the first author was supported by a NSERC grant.

We would like to thank referees for a very detailed report that improved the paper.

\section{References}

[1] Avram, F. and Taqqu, M. S. (1987) Noncentral limit theorems and Appell polynomials. Annals of Probability 15, 767-775.

[2] Csörgö, S. and Mielniczuk, J. (1995) Density estimation under long-range dependence. Annals of Statistics 23, 990-999.

[3] Davydov, Ju. A. (1970) The invariance principle for stationary processes. Teor. Verojatnost. i Primenen. 15, 498-509.

[4] Fan, J. and Yao, Q. (1998) Efficient estimation of conditional variance functions in stochastic regression. Biometrika 85, 645-660.

[5] Guo, H. and Koul, H. L. (2008) Asymptotic inference in some heteroscedastic regression models with long memory design and errors. Annals of Statistics $36,458-487$.

[6] Kulik, R. and Lorek, P. (2011) Some results on random design regression with long memory errors and predictors. Journal of Statistical Planning and Inference 141, 508-523. 
[7] Kulik, R. and Wichelhaus, C. (2011) Nonparametric conditional variance and error density estimation in regression models with dependent errors and predictors. Electronic Journal of Statistics 5, 856-898.

[8] Lahiri, S. N. (2003) Resampling methods for dependent data. Springer Series in Statistics.

[9] Mielniczuk, J. and Wu, W. B. (2004) On random-design model with dependent errors. Statistica Sinica 14, 1105-1126.

[10] Taqqu, M. S. (2003) Fractional Brownian motion and long-range dependence. Theory and applications of long-range dependence, 5-38. Birkhäuser.

[11] Wang, L., Brown, L. D., Cai, T. T. and Levine, M. (2008) Effect of mean on variance function estimation in nonparametric regression. Annals of Statistics 36, 646-664.

[12] Wu, W. B. and Mielniczuk, J. (2002) Kernel density estimation for linear processes. Annals of Statistics 30, 1441-1459.

[13] Zhao, Z. and Wu, W. B. (2008) Confidence bands in nonparametric time series regression. Annals of Statistics 36, 1854-1878. 